\title{
Alveolar macrophages modulate allergic inflammation in a murine model of asthma
}

\author{
Bo-Ram Bang ${ }^{1,2}$, Eunyoung Chun ${ }^{1,2}$, \\ Eun-Jin Shim ${ }^{1,2}$, Hyun-Seung Lee ${ }^{1,2}$, \\ Soo-Yeon Lee ${ }^{1,2}$, Sang-Heon Cho ${ }^{1,2}$, \\ Kyung-Up Min ${ }^{1,2}$, You-Young Kim ${ }^{1,2}$ \\ and Heung-Woo Park ${ }^{1,2,3}$ \\ ${ }^{1}$ Department of Internal Medicine \\ ${ }^{2}$ Institute of Allergy and Clinical Immunology \\ College of Medicine \\ Seoul National University \\ Seoul 110-744, Korea \\ ${ }^{3}$ Corresponding author: Tel, 82-2-2072-0699; \\ Fax, 82-2-742-2912; E-mail, guinea71 @snu.ac.kr \\ DOI 10.3858/emm.2011.43.5.028 \\ Accepted 18 March 2011 \\ Available Online 18 March 2011
}

Abbreviations: AM, alveolar macrophage; BAL, bronchoalveolar lavage; LPS, lipopolysaccharide; OVA, ovalbumin

\begin{abstract}
The role of alveolar macrophages (AMs) in the pathogenesis of asthma is still unknown. The aim of the present study was to investigate the effects of $A M$ in the murine model of asthma. AMs were selectively depleted by liposomes containing clodronate just before allergen challenges, and changes in inflammatory cells and cytokine concentrations in bronchoalveolar lavage (BAL) fluid were measured. AMs were then adoptively transferred to AM-depleted sensitized mice and changes were measured. Phenotypic changes in AMs were evaluated after in vitro allergen stimulation. AM-depletion after sensitization significantly increased the number of eosinophils and lymphocytes and the concentrations of IL-4, IL-5 and GM-CSF in BAL fluid. These changes were significantly ameliorated only by adoptive transfer of unsensitized AMs, not by sensitized AMs. In addition, in vitro allergen stimulation of AMs resulted in their gaining the ability to produce inflammatory cytokines, such as IL-1 $\beta$, IL-6 and TNF- $\alpha$, and losing the ability to suppress GM-CSF concentrations in BAL fluid. These findings suggested that AMs worked probably through GM-CSF-dependent mechanisms, although further confirmatory experiments are needed. Our results indicate that the role
\end{abstract}

\author{
of AMs in the context of airway inflammation should be \\ re-examined.
}

\section{Keywords:}

adoptive transfer; airway inflammation; alveolar macrophage; asthma; GM-CSF

\section{Introduction}

Alveolar macrophages (AMs) are the most abundant cells in the alveolar spaces and conducting airways, and are known to be involved in immune homeostasis in the respiratory tract (Wissinger et al., 2009). Asthma is a disease characterized by immune-mediated airway inflammation. Several reports have suggested that AMs suppress allergenspecific immune response and airway inflammation (Thepen et al., 1991, 1992; Tang et al., 2001; Careau et al., 2010). However, this property seems to be dependent on the functional status of AMs. Sensitized AMs promote eosinophilic airway inflammation (Moon et al., 2007) and take part in acute asthma exacerbation by stimulating CD4-positive $\mathrm{T}$ cells to secrete cytokines (Herbert et al., 2010). A recent report has shown that allergen sensitization modulates $A M$ function and only unsensitized AMs protect against development of airway hyperresponsiveness (Careau et al., 2006).

In this study, we compared the effects of sensitized and unsensitized AMs on the reduction of allergic inflammation and cytokine secretion in a murine model of asthma. Phenotype changes were first evaluated after selective depletion of AM populations with liposomes containing clodronate (dichloromethylenediphosphate or $\mathrm{Cl}_{2} \mathrm{MDP}$ ). Then, sensitized or unsensitized AMs were adoptively transferred into AM-depleted sensitized mice and numbers of inflammatory cells and cytokine concentrations in bronchoalveolar (BAL) fluid were measured. Finally, we evaluated the phenotypic changes in AMs after in vitro allergen stimulation.

\section{Results}

Changes in inflammatory cells and cytokine levels in BAL fluid after alveolar macrophage depletion

Administration of clodronate $\left(\mathrm{Cl}_{2} \mathrm{MDP}\right)$-encapsulated 
liposomes resulted in significant increases in total cell, eosinophil, and lymphocyte counts in BAL fluid (Figure 1). Cytokine concentrations in BAL fluid, IL-5 and GM-CSF, showed significant increases after AM-depletion (Figure 2). IFN- $\gamma$ and IL-13 (data not shown) concentrations showed no differences and the levels of IL-12p70 were below the detection limit.

\section{Changes in inflammatory cells and cytokine levels in BAL fluid after adoptive transfer of sensitized or unsensitized alveolar macrophages to alveolar macrophage depleted sensitized mice}

When sensitized AMs were adoptively transferred to AM-depleted sensitized mice, there were no differences in BLA fluid inflammatory cell counts. However, adoptive transfer of unsensitized AMs significantly reduced total cell, eosinophil, and lymphocyte counts in BAL fluid (Figure 1). Similarly, adoptive transfer of sensitized AMs did not significantly decrease the concentrations of inflammatory cytokines in BAL fluid. However, adoptive transfer of unsensitized AMs significantly reduced the concentrations of GM-CSF in BAL fluid (Figure 2). Macrophages recruited from regional tissue or circulation (Moon et al., 2007) after intranasal challenge of OVA may explain the insignificant difference in the number of macrophages shown in Figure 1.

\section{Pro-inflammatory cytokine production from alveolar macrophages after in vitro OVA stimulation}

AMs obtained from unsensitized mice expressed little or nopro-inflammatory cytokines (IL-1 $\beta$, IL-6, and TNF- $\alpha$ ), but AMs obtained from sensitized mice produced significantly higher concentrations of pro-inflammatory cytokines after in vitro OVA stimulation (Figure 3).

\section{Discussion}

In the present study, we demonstrated that AMdepletion after sensitization significantly increased the number of eosinophilis and lymphocytes, and the concentrations of IL-4, IL-5, and GM-CSF in $\mathrm{BAL}$ fluid in a murine model of asthma. Moreover, increased numbers of eosinophilis and lymphocytes and concentrations of GM-CSF in BAL fluid after AM-depletion were significantly ameliorated
A

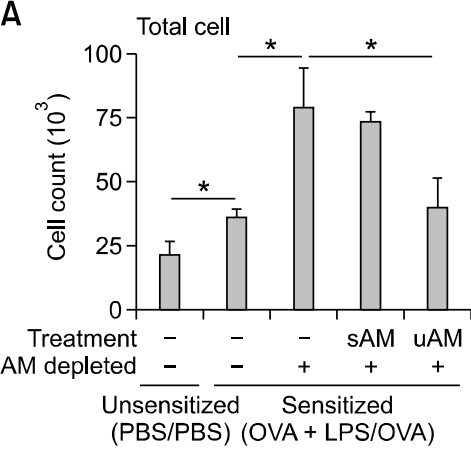

C

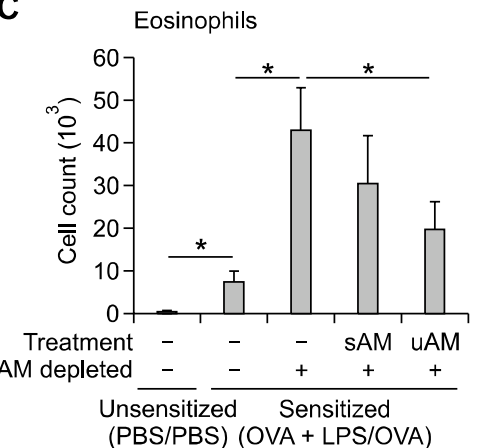

B

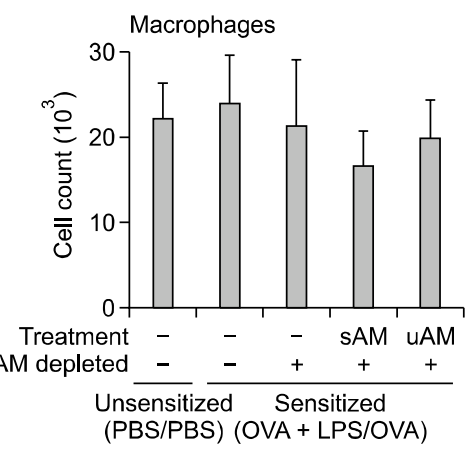

D

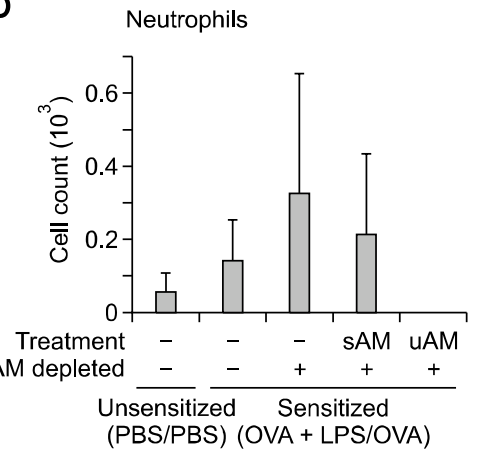

$E$

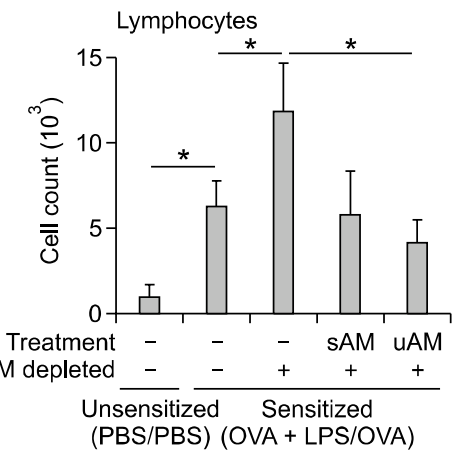

Figure 1. Changes in inflammatory cells in BAL fluid after alveolar macrophage depletion and after adoptive transfer of sensitized or unsensitized alveolar macrophage to alveolar macrophage depleted mice (A) total cell, (B) macrophages, (C) eosinophils, (D) neutrophils, (E) lymphocytes, LPS, lipopolysaccharide; sAM, sensitized alveolar macrophages; uAM, unsensitized alveolar macrophages; OVA, ovalbumin, $\left.{ }^{*}, P<0.05\right)$ 
A

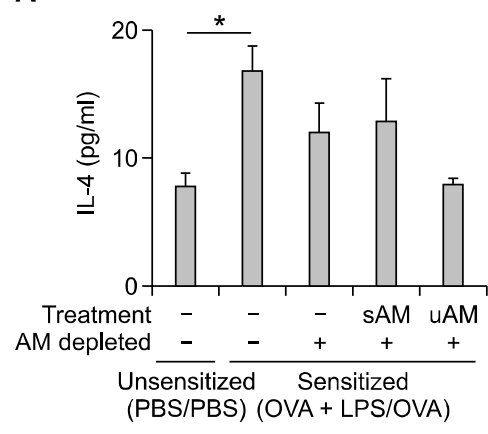

C

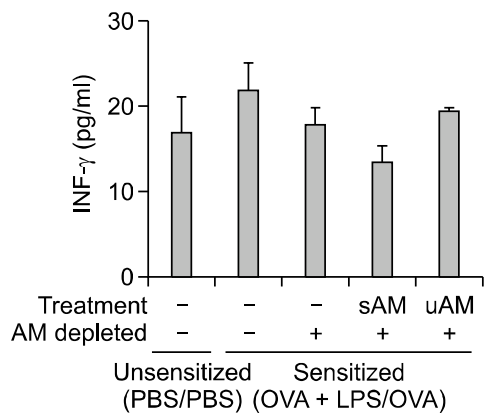

B

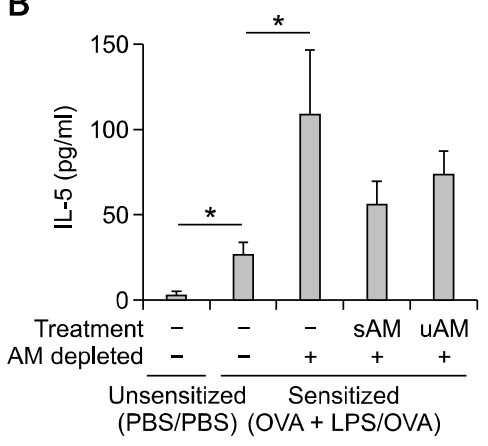

D

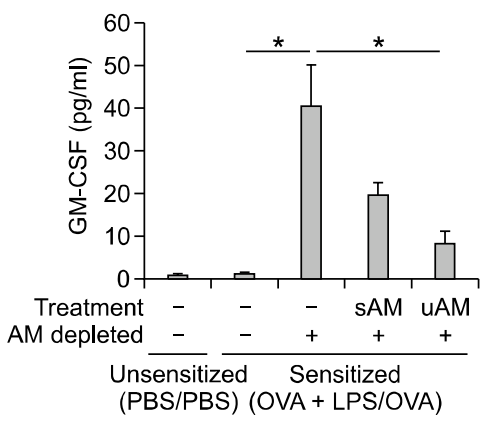

Figure 2. Changes in inflammatory cytokine levels in BAL fluid after alveolar macrophage depletion and after adoptive transfer of sensitized or unsensitized alveolar macrophage to alveolar macrophage depleted mice (A, IL-4; B, IL-5; C, IFN- $\gamma$; D, GM-CSF; LPS, lipopolysaccharide; SAM, sensitized alveolar macrophages; UAM, unsensitized alveolar macrophages; OVA, ovalbumin; * $P<0.05$ )

only by adoptive transfer of unsensitized AMs, not by sensitized AMs. The observations, in conjunction with a previous report that unsensitized AMs can protect against development of airway hyperresponsiveness (Careau et al., 2006), suggest that AMs may have an important role in the development of asthma. In addition, we believe that the gain in ability to produce inflammatory cytokines, such as IL-1 $\beta$, IL- 6 and TNF- $\alpha$, and the loss of ability to suppress GM-CSF concentrations in BAL fluid may contribute to the inability of sensitized AMs to play a protective role.

It was suggested that upon exposure to allergen, lung macrophages can convert to a Th1-oriented antigen presenting capacity by an IFN- $\gamma$-dependent mechanism, and that the participation of these cells in antigen presentation inhibits, to some degree, the development of Th2 immune responses in the bronchial mucosa (Tang et al., 2001). Along with previous reports, we found that populations of eosinophils and lymphocytes in BAL fluid significantly increased after AM-depletion. However, we could not observe any significant change in IFN- $\gamma$ concentrations in BAL fluid after AM-depletion, whereas, the GM-CSF concentrations in BAL fluid

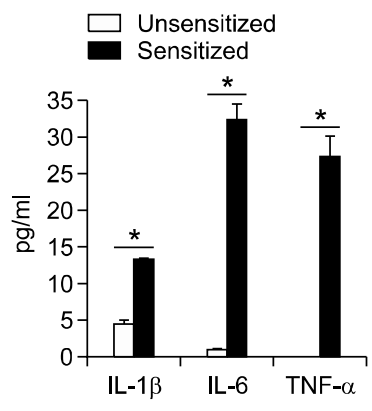

Figure 3. Pro-inflammatory cytokine production from alveolar macrophages after in vitro ovalbumin stimulation $\left({ }^{*}, P<0.05\right)$.

changed significantly. This different observation may have been a result of differences in the mouse strain used and the experimental protocol, and detailed mechanisms for these findings should be investigated further. To the best of our knowledge, this is the first study showing that AMs may modulate allergic inflammation via GM-CSF in a murine model of asthma.

A recent report showed that the transfer of unsensitized AMs $24 \mathrm{~h}$ prior to OVA challenge 
administered to AM-depleted, sensitized rats resulted in the abrogation of airway hyperresponsiveness, whereas the transfer of sensitized AMs to AMdepleted, unsensitized rats did not significantly alter airway responsiveness (Careau et al., 2006). However, the authors did not observe any differences in the numbers of inflammatory cells in BAL fluid after AM depletion and transfer and concluded that the role of AMs in asthma is unrelated to reduction in inflammatory cell recruitment, but instead may involve other mechanisms modulating airway hyperresponsiveness. The observations are contrary to our findings. However, airway hyperresponsiveness generally correlates reasonably well with airway inflammation (Busse and Lemanske, 2001). AMs have long been recognized to be activated in asthmatic inflammation, with evidence of increased secretion of cytokines such as tumor necrosis factor a and IL-6 (Gosset et al., 1991). In addition, it has been revealed that AMs from atopic asthmatic subjects, but not from atopic non-asthmatic subjects, enhance IL-4 and IL-5 production by $\mathrm{CD} 4^{+} \mathrm{T}$ cells upon stimulation with allergen (Tang et al., 1998a, 1998b). Recently, it was reported that the airway epithelial cell derived IL-33 amplified an alternative activation and chemokine productions from macrophages (KurowskaStolarska et al., 2009). Taken together, it is clear that AM plays a potential role in the modulation of local inflammation.

However, in the present study using a murine model of asthma, we found that airway inflammation was not inhibited and even was increased after AM depletion. Similar findings were observed, in a rat model of trimellitic anhydride-induced occupational asthma (Valstar et al., 2006). The authors reported that AM depletion augmented the trimellitic anhydride-induced tissue damage and inflammation $24 \mathrm{~h}$ after challenge. The reason for this phenomenon could be based on the functional status of the AMs. As mentioned previously, AMs play a key role in the maintenance of immunological homeostasis in the respiratory tract. As examples, allergen challenge of AM-depleted sensitized rats results in a highly elevated immunoglobulin $E$ concentrations, large influxes of $T$ and $B$ cells into the lungs, and increase in airway hyperresponsiveness (Thepen et al., 1991, 1992; Careau et al., 2006). We also observed that inflammatory cells in BAL fluid significantly increased when AMs were depleted in mice in a murine model of asthma, and these increases were attenuated by the adoptive transfer of AMs, although significant changes were found only when unsensitized AMs were transferred. This suggest that unsensitized AMs are capable of reducing allergic inflammation, but they lose this ability as they become sensitized after repeated exposures to allergen. The inflammatory phenotypic that we observed in the present study maybe one of the reasons for this finding.

In summary, AM depletion significantly increased the numbers of total inflammatory cells, eosinophils and lymphocytes in BAL fluid, and adoptive transfer of unsensitized AMs effectively abrogated these change, probably through GM-CSF dependent mechanisms. Our results indicate the role of AMs in the context of airway inflammation should be re-examined.

\section{Methods}

\section{Mice}

Female C57BL/6 mice (6-week old) were purchased from the Jackson Laboratory (Bar Harbor, ME). All animals were maintained in filter-top cages under specific pathogen-free conditions at the animal facility of the Seoul National University College of Medicine (Seoul, Korea). Experimental procedures were performed with the approval of the Seoul National University Institutional Animal Care and Use Committee (IACUC) in accordance with the guidelines of IACUC.

\section{Experimental protocols for a murine model of asthma and alveolar macrophage depletion}

For sensitization, mice were anesthetized and ovalbumin (OVA, Sigma Chemical Co., St. Louis, US) plus $0.1 \mu \mathrm{g}$ of lipopolysaccharide (E. coli O55:B5, Calbiochem, San Diego, US) in $30 \mu \mathrm{l}$ of phosphate buffered saline (PBS) were instilled intranasally on days $1,2,3$, and 8 . On days 7 and 8 after the last sensitization with OVA plus LPS (days 15, 16), mice were intranasally challenged with OVA only (Supplemental Data Figure S1A). For AM depletion, mice were first challenged with OVA plus LPS as described previously (Elder et al., 2004), and then received $30 \mu \mathrm{l}$ of clodronate $\left(\mathrm{Cl}_{2} \mathrm{MDP}\right)$-encapsulated liposomes (Roche Diagnostics $\mathrm{GmbH}$, Mannheim, Germany) on 3 consecutive days (days 12-14) just before the first OVA challenge (Supplemental Data Figure S1B). Confirmation that more than $75 \%$ of alveolar macrophages were depleted without affecting other cell types were performed by counting the macrophages in BAL fluid from treated and untreated mice.

\section{Isolation of alveolar macrophages and adoptive transfer}

Sensitized AMs were obtained from lungs of sacrificed mice 7 days after the last sensitization (day 15 in Supplemental Data Figure S1A), and unsensitized AMs were obtained from lungs of sacrificed mice who did not undergo sensitization. The lungs were perfused via the right ventricle with $5 \mathrm{ml}$ of PBS and excised and minced lung tissue was incubated for $15 \mathrm{~min}$ at $37^{\circ} \mathrm{C}$ in $0.5 \%$ EDTA. To 
obtain a single cell, the minced tissue was grinded on the strainer with complete RPMI (cRPMI) that was supplemented $10 \%$ FBS, L-glutamine, sodium pyruvate, penicillin-streptomycin, and erythrocytes were lysed by treatment with ammonium chloride solution (StemCell technologies, Vancouver, Canada). The cells were washed twice with RPMI, and centrifuged at $1500 \mathrm{rpm}$ for $10 \mathrm{~min}$. We defined AMs as

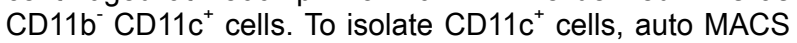
sorter (Miltenyi Biotec, Auburn, CA) with anti-CD11c antibody conjugated to magnetic beads were used in accordance with manufacturer's instructions. Isolated $\mathrm{CD}_{11 \mathrm{c}^{+}}$ cells were then stained with anti-CD11b to sort CD11 ${ }^{-}$ $\mathrm{CD}_{11 \mathrm{c}^{+}}$cells. The stained cells were gated into CD11 $\mathrm{b}^{-}$ $\mathrm{CD}_{11 \mathrm{c}^{+}}$cells according to expressions of CD11b or CD11c molecule and sorted using the live sterile cell sorting system (FACSAria system, Becton Dickinson, San Jose,

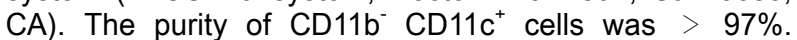
Isolated sensitized or unsensitized AMs $\left(1 \times 10^{5}\right.$ cells $)$ were transferred intratracheally to AM-depleted sensitized mice on the day of the first OVA challenge (day 15, Supplemental Data Figure S1C). Mice were sacrificed on day 18 , and lung inflammation was evaluated (Supplemental Data Figure S1C).

\section{Bronchial alveolar lavage fluid collection and cytokine measurements}

A total of $2 \mathrm{ml}(1 \mathrm{ml} \times 2)$ of BAL fluid using PBS was obtained after tracheostomy. Cells were collected by centrifugation at $3000 \mathrm{rpm}$ for $15 \mathrm{~min}$ at $4^{\circ} \mathrm{C}$. The pellets were resuspended in $100 \mu \mathrm{l}$ of PBS, and total cells counts were determined using a Neubauer's chamber (hemocytometer). To determine differential cell counts, cytocentrifugation (Cytospin 3, Shandon, UK) was used at $500 \mathrm{rpm}$ for $5 \mathrm{~min}$ at room temperature. BAL cells on slides were stained with Diff-Quik (Sysmex Co., Japan) and after airdry, fixed with a synthetic mounting medium (Histomount, Ted Pella Inc.). At least 300 cells were counted in each preparation. The numbers of macrophages, eosinophils, neutrophils and lymphocytes in BAL fluid were determined as described previously (Moon et al., 2010). The concentrations of IL-12p70, IFN- $\gamma$, IL-4, IL-5, IL-13 and GM-CSF in BAL fluids were measured using the Bio-Plex cytokine assay in accordance with the manufacturer's instructions (Bio-Rad Laboratories, Inc., Hercules, CA). Briefly, prepared beads were added to the pre-wetted filter plate, and then samples were added and incubated for $30 \mathrm{~min}$ at room temperature. Attached cytokines were detected by incubating the plates for 30 min with detection antibodies. Streptavidin-PE was added to the filter plates, which were read on the Bio-Plex reader.

\section{Characterization of sensitized and unsensitized alveolar macrophages}

To compare sensitized AMs and unsensitized AMs, proinflammatory cytokines were evaluated after in vitro stimulation of the cells by OVA. AMs (CD11 $b^{-}$CD11 ${ }^{+}$cells) were isolated from unsensitized and sensitized mice (just before OVA challenge; day 15 in Supplemental Data Figure S1A). The cells were stimulated by OVA $(100 \mu \mathrm{g} / \mathrm{ml})$ for 3 days, and then the concentrations of IL- $1 \beta$, IL- 6 and
TNF- $\alpha$ in cell culture supernatant were measured using the Bio-Plex cytokine assay.

\section{Statistical analysis}

All data are reported as the mean \pm standard error (SE) of the means. A two-tailed Student $t$-test was used for analysis and differences were considered significant for $P$ $<0.05$.

\section{Supplemental data}

Supplemental data include a figure and can be found with this article online at http://e-emm.or.kr/article/article_files/ SP-43-5-02.pdf.

\section{Acknowledgements}

This work was supported by a grant of the Korea Healthcare technology R\&D Project, from Ministry of Health and Welfare, Republic of Korea (A102065), and by a grant from Seoul National University Hospital (0420070550).

\section{References}

Busse WW, Lemanske RF Jr. Asthma. N Engl J Med 2001; 344:350-62

Careau E, Proulx LI, Pouliot P, Spahr A, Turmel V, Bissonnette EY. Antigen sensitization modulates alveolar macrophage functions in an asthma model. Am J Physiol Lung Cell Mol Physiol 2006;290:L871-9

Careau E, Turmel V, Lauzon-Joset JF, Bissonnette EY. Alveolar macrophages reduce airway hyperresponsiveness and modulate cytokine levels. Exp Lung Res 2010;36: 255-61

Elder AC, Gelein R, Oberdorster G, Finkelstein J, Notter R, Wang Z. Efficient depletion of alveolar macrophages using intratracheally inhaled aerosols of liposome-encapsulated clodronate. Exp Lung Res 2004;30:105-20

Gosset P, Tsicopoulos A, Wallaert B, Vannimenus C, Joseph $M$, Tonnel $A B$, Capron $A$. Increased secretion of tumor necrosis factor alpha and interleukin- 6 by alveolar macrophages consecutive to the development of the late asthmatic reaction. J Allergy Clin Immunol 1991;88:561-71

Herbert C, Scott MM, Scruton KH, Keogh RP, Yuan KC, Hsu K, Siegle JS, Tedla N, Foster PS, Kumar RK. Alveolar macrophages stimulate enhanced cytokine production by pulmonary CD4+ T-lymphocytes in an exacerbation of murine chronic asthma. Am J Pathol 2010;177:1657-64

Kurowska-Stolarska M, Stolarski B, Kewin P, Murphy G, Corrigan CJ, Ying S, Pitman N, Mirchandani A, Rana B, van Rooijen N, Shepherd M, McSharry C, Mclnnes IB, Xu D, Liew FY. IL-33 amplifies the polarization of alternatively activated macrophages that contribute to airway inflammation. J Immunol 2009;183:6469-77

Moon HG, Kim YS, Choi JP, Choi DS, Yoon CM, Jeon SG, 
Gho YS, Kim YK. Aspirin attenuates the anti-inflammatory effects of theophylline via inhibition of cAMP production in mice with non-eosinophilic asthma. Exp Mol Med 2010; 42:47-60

Moon KA, Kim SY, Kim TB, Yun ES, Park CS, Cho YS, Moon HB, Lee KY. Allergen-induced CD11b+ CD11c(int) CCR3+ macrophages in the lung promote eosinophilic airway inflammation in a mouse asthma model. Int Immunol 2007; 19:1371-81

Tang C, Rolland JM, Li X, Ward C, Bish R, Walters EH. Alveolar macrophages from atopic asthmatics, but not atopic nonasthmatics, enhance interleukin-5 production by CD4+ T cells. Am J Respir Crit Care Med 1998a;157:1120-6

Tang C, Rolland JM, Ward C, Thien F, Li X, Gollant S, Walters $\mathrm{EH}$. Differential regulation of allergen-specific $\mathrm{T}(\mathrm{H} 2)$ - but not $\mathrm{T}(\mathrm{H} 1)$-type responses by alveolar macrophages in atopic asthma. J Allergy Clin Immunol 1998b;102:368-75

Tang C, Inman MD, van Rooijen N, Yang $P$, Shen $H$, Matsumoto K, O'Byrne PM. Th type 1-stimulating activity of lung macrophages inhibits Th2-mediated allergic airway inflammation by an IFN-gamma-dependent mechanism. J Immunol 2001;166:1471-81

Thepen T, McMenamin C, Oliver J, Kraal G, Holt PG. Regulation of immune response to inhaled antigen by alveolar macrophages: differential effects of in vivo alveolar macrophage elimination on the induction of tolerance vs. immunity. Eur J Immunol 1991;21:2845-50

Thepen T, McMenamin C, Girn B, Kraal G, Holt PG. Regulation of IgE production in pre-sensitized animals: in vivo elimination of alveolar macrophages preferentially increases IgE responses to inhaled allergen. Clin Exp Allergy 1992;22:1107-14

Valstar DL, Schijf MA, Nijkamp FP, Storm G, Arts JH, Kuper $\mathrm{CF}$, Bloksma N, Henricks PA. Alveolar macrophages have a dual role in a rat model for trimellitic anhydride-induced occupational asthma. Toxicol Appl Pharmacol 2006;211: 20-9

Wissinger E, Goulding J, Hussell T. Immune homeostasis in the respiratory tract and its impact on heterologous infection. Semin Immunol 2009;21:147-55 